\title{
Masculinization of the X-chromosome in aphid soma and gonads
}

Jaquiéry $\mathrm{J}^{*}, 1$, Simon $\mathrm{J}^{-\mathrm{C}^{1}}$, Robin $\mathrm{S}^{1,2}$, Richard $\mathrm{G}^{1}$, Peccoud $\mathrm{J}^{3}$, Boulain $\mathrm{H}^{4}$, Legeai $\mathrm{F}^{1,2}$, Tanguy $\mathrm{S}^{1}$, Prunier-Leterme $\mathrm{N}^{1}$, Le Trionnaire $\mathrm{G}^{1}$

${ }^{1}$ INRAE, UMR1349, Institute of Genetics, Environment and Plant Protection, Le Rheu, France

${ }^{2}$ University of Rennes, Inria, CNRS, IRISA F-35000 Rennes, France

${ }^{3}$ Laboratoire Ecologie et Biologie des Interactions, Equipe Ecologie Evolution Symbiose,

Unité Mixte de Recherche 7267 Centre National de la Recherche Scientifique, Université de Poitiers, 86073 Poitiers CEDEX 9, France

${ }^{4}$ Department of Ecology and Evolution, University of Lausanne, 1015, Lausanne, Switzerland

*Correspondence: Julie Jaquiéry, Julie.Jaquiery@inrae.fr

Keywords: Sex-biased gene expression, sexual conflict, sexual antagonism, dimorphism, sex chromosome, duplication 


\section{Summary}

Males and females share essentially the same genome but differ in their optimal values for many phenotypic traits, which can result in intra-locus conflict between the sexes. Aphids display XX/X0 sex chromosomes and combine unusual $X$ chromosome inheritance with cyclical parthenogenesis. Theoretical and empirical works support the hypothesis that the large excess of male-biased genes observed on the aphid X chromosome compared to autosomes has evolved in response to sexual conflicts, by restricting the product of a sexually antagonistic allele to the sex it benefits. However, whether such masculinization of the $\mathrm{X}$ affects all tissues (as expected if it evolved in response to sexual conflicts) or reflects tissue specificities (which would contradict the sexual conflict hypothesis) remains an open question. To address it, we measured gene expression in different somatic and gonadic tissues of males, sexual females and parthenogenetic females of the pea aphid. We observed a masculinization of the $X$ at the tissue-level, with male-biased genes being 2.5 to 3.5 more frequent on the $X$ than expected. We also tested the hypothesis that gene duplication can facilitate the attenuation of conflicts by allowing gene copies to neo- or subfunctionalize and reach sex-specific optima. As predicted, X-linked copies of duplicated genes having their other copies on autosomes were more frequently male-biased $(40.5 \%$ of the genes) than duplicated autosomal genes (6.6\%) or X-linked single-copy genes (32.5\%). These results highlight a peculiar pattern of expression of X-linked genes in aphids at the tissue level and provides further support for sex-biased expression as a mechanism to attenuate intra-locus sexual conflicts. 


\section{Introduction}

Sexual dimorphism, the difference between males and females at any phenotypic trait such as behavior, morphology, physiology or life history, is widespread. These differences are pervasive among eukaryotes, from plants to nematodes, insects, birds and mammals, to name a few (Cox and Calsbeek 2009; Williams and Carroll 2009). Regardless of its amount, sexual dimorphism engages males and females in a constant tug-of-war because their reproductive interests (such as optimal mating rate, number of partners, parental investment, ...) never align, owing to constitutive investment differences in gametes and/or progeny (Bonduriansky and Chenoweth 2009).

Differences in optimal trait values between sexes may generate intra-locus sexual conflicts. Typically, a new allelic variant could be beneficial to a female but deleterious to a male or vice versa. Such a sexually antagonistic (SA) allele is predicted to increase in frequency as long as the cost/benefit balance is positive. This increase leads to a so-called gender load in the population, due to the transmission of SA alleles to both sons and daughters (Chippindale et al. 2001; Rice and Chippindale 2002; Bonduriansky and Chenoweth 2009).

Several mechanisms may alleviate gender load (Bonduriansky and Chenoweth 2009). One is the evolution of sex-biased or sex-specific gene expression through a modifier of expression (Rice 1984). Once a SA allele is frequent enough, the reduction of its expression in the sex where it is deleterious may allow this variant to further increase in frequency and possibly reach fixation (Rice 1984; Ellegren and Parsch 2007; Bonduriansky and Chenoweth 2009). This implies that the reduction of expression of the SA allele is beneficial to individuals of this sex. For genes that must be expressed at a certain level, a gene duplication event could allow bringing a new gene copy to sub- or neo-functionalize toward the sex-specific optimum (Bonduriansky and Chenoweth 2009; Connallon and Clark 2011; Gallach and Betrán 2011). Interestingly, these two processes (the duplication and the change in expression) could occur simultaneously, when the duplicated copy inserts in a region of the genome that already shows specific expression pattern (e.g., Arthur et al. 2014). 
The invasion of the population by a SA allele and the attenuation of gender load through duplication and/or evolution of sex-biased gene expression may take place at different timescales. Indeed, the increase in frequency of a SA allele can be as rapid as a few generations, depending on its effect on fitness (e.g., Dean et al. 2012 for an experimental demonstration). The attenuation of the conflict by expression change or gene duplication may take much longer as it relies on rare random events, themselves depending on effective population size and mutation rate (Rice 1984; Stewart et al. 2010; Connallon and Clark 2011; Collet et al. 2016).

Importantly, the conditions for invasion by a SA allele differ between autosomes and sex chromosomes (Rice 1984; Fry 2010). In XX/XY (and ZZ/ZW) systems, any SA allele that benefits males can invade the $Y$ without conflict (the same is true for female-beneficial alleles on the $\mathrm{W}$ ) assuming complete linkage between the SDR (sex-determining region) and the SA locus. The picture for the X (or Z) is more complex (Vicoso and Charlesworth 2006). Xlinked recessive alleles are exposed to selection in males, while the female-biased transmission of the $\mathrm{X}$ ( $\mathrm{X}$ chromosomes are transmitted twice more often by females than by males) gives more importance to selection episodes occurring in females. As a result, the $X$ should accumulate recessive male-beneficial alleles and dominant female-beneficial ones. Similar processes are expected to occur in ZZ/ZW systems (e.g., birds, lepidopterans...).

Aphids constitute an interesting model to study the evolution of SA alleles as they show an $\mathrm{XX} / \mathrm{XO}$ sex-determining system combined with cyclical parthenogenesis: the alternation between several parthenogenetic generations in spring and summer and a single sexual generation in autumn. As a result, three distinct reproductive morphs occur in aphids: males, sexual females and parthenogenetic (asexual) females. Sexual females are genetically identical to their parthenogenetic mother, while male production involves the random elimination of one of the $X$ (Wilson et al. 1997). Furthermore, during spermatogenesis only sperm cells carrying an X chromosome develop (Blackman 1987), so that the fusion of a sperm cell $(A X)$ and an ovum (AX) produces exclusively a diploid individual at the $\mathrm{X}$ and autosomes, which develops into a parthenogenetic female.

Theoretical models (Jaquiéry et al. 2013) predict that the peculiar inheritance of the $\mathrm{X}$ in aphids, the alternation between sexual and asexual reproduction, and the presence of 
three different morphs (sexual females, parthenogenetic females and males) have a major influence on the genomic location of SA allelic variants. In particular, conditions for the invasion of variants that are beneficial to males and deleterious to parthenogenetic females are predicted to be less restrictive for the $X$ than for autosomes. By contrast, the conditions for the invasion of variants that are detrimental to males and beneficial for parthenogenetic females are more restrictive for the $X$. These models thus predict the $X$ to be optimized for male functions. Genomic analyses on the pea aphid Acyrthosiphon pisum showed that the $\mathrm{X}$ chromosome had a large excess of genes preferentially expressed in males (i.e., male-biased genes) compared to autosomes, and a deficit of parthenogenetic female-biased genes, resulting in a "masculinization" of this chromosome (Jaquiéry et al. 2013). This pattern matched predictions made under the hypothesis that evolution of sex-biased gene expression reduces sexual conflicts by decreasing the expression of a sexually antagonistic allele to the sex it benefits (Rice 1984). Interestingly, masculinization of the $X$ has also been observed in another aphid species (Myzus persicae) that diverged from the pea aphid lineage 40 MYA (million years ago) (Mathers et al. 2019), but not in psyllids (Li et al. 2020) an obligatory group of sexual species closely related to aphids. These studies provide further support that the masculinization of the $\mathrm{X}$ evolved in response to intra-locus sexual conflicts resulting from the peculiar life cycle (cyclical parthenogenesis) and $\mathrm{X}$ inheritance in aphids. However, as previous studies on aphids analyzed whole-body transcriptomes (Jaquiéry et al. 2013; Jaquiéry et al. 2018; Mathers et al. 2019; Li et al. 2020), it remains unclear whether the observed masculinization of the $X$ systematically occurs within each type of tissue or is driven by some specific tissue with unusual expression patterns. For example, a metaanalysis of gene expression in Drosophila revealed that the $\mathrm{X}$ is enriched in male-biased genes in the brain, while for all other tissues there is either no significant excess or a paucity of male-biased genes on this chromosome (Huylmans et al. 2015). Tissue-specific patterns may thus originate from the interplay between sex-specific regulation of expression (including sexual antagonism, a force presumably acting in all tissues), and dosage compensation, which could be stronger in the brain (Nozawa et al. 2013, Vensko and Stone 2015).

Here, we predicted that if masculinization of the aphid X chromosome evolved solely in response to intra-locus sexual conflicts, masculinization would occur in all tissues. To 
verify this prediction, we measured gene expression in different tissues from males, sexual females and parthenogenetic females, including gonadic and somatic tissues. We found a masculinization of the $\mathrm{X}$ in each type of tissue. Moreover, we confirmed that the $\mathrm{X}$-linked copy of a duplicated gene (having another copy on autosomes) is more likely to show a male-biased expression than its autosomal copy or an X-linked single copy gene. This result suggests that duplications facilitate sub- or neo-functionalization toward the sex-specific optimum.

\section{Results}

\section{Gene expression levels}

Gene expression levels in three different tissues (heads, legs, gonads) of the three morphs (males, sexual females, parthenogenetic females) were measured from RNA-seq counts on individuals produced by the same pea aphid clonal lineage (Supplementary table S1). Overall, 14,605 genes out of the 20,639 predicted genes were expressed ( $>1$ count per million reads [CPM] in at least two samples) in the 18 samples ( 3 morphs $\times 3$ tissues $\times 2$ replicates). We assigned $18,719(90.7 \%)$ of the 20,639 predicted genes as autosomal or Xlinked, based on scaffold assignments from Jaquiéry et al. (2018). The genes that were not assigned $(9.3 \%)$ were located on scaffolds or part of scaffolds that were not clearly assigned to $X$ or autosomes in Jaquiéry et al. (2018) and they were thus not considered in the subsequent analyses. Only 51\% (3044/5961) of the X-linked genes were found to be expressed, against $85 \%(10,890 / 12,758)$ of the autosomal genes (figure $1 \mathrm{~A})$. On average, more genes were expressed in male samples than in female samples, especially for X-linked genes (X-chromosome: median number of expressed genes in males $=2020$, median in females $=1425$, two-sided Mann-Whitney test, $p=0.0009 ;$ autosomes: median in males = 9061, median in females $=8560$, two-sided Mann-Whitney test, $p=0.067$, figure 1B).

In the heatmap based on gene expression levels, samples grouped systematically by replicate of the same biological condition, and then by tissue for leg and head samples (figure 1C, supplementary figure S1). Within each of these tissues, the four female samples 
were always more similar to each other than to the male samples. Gonad samples were the most heterogeneous ones, samples from testes (MG) being highly different from all other samples, and samples from parthenogenetic and sexual female gonads grouping together.

A
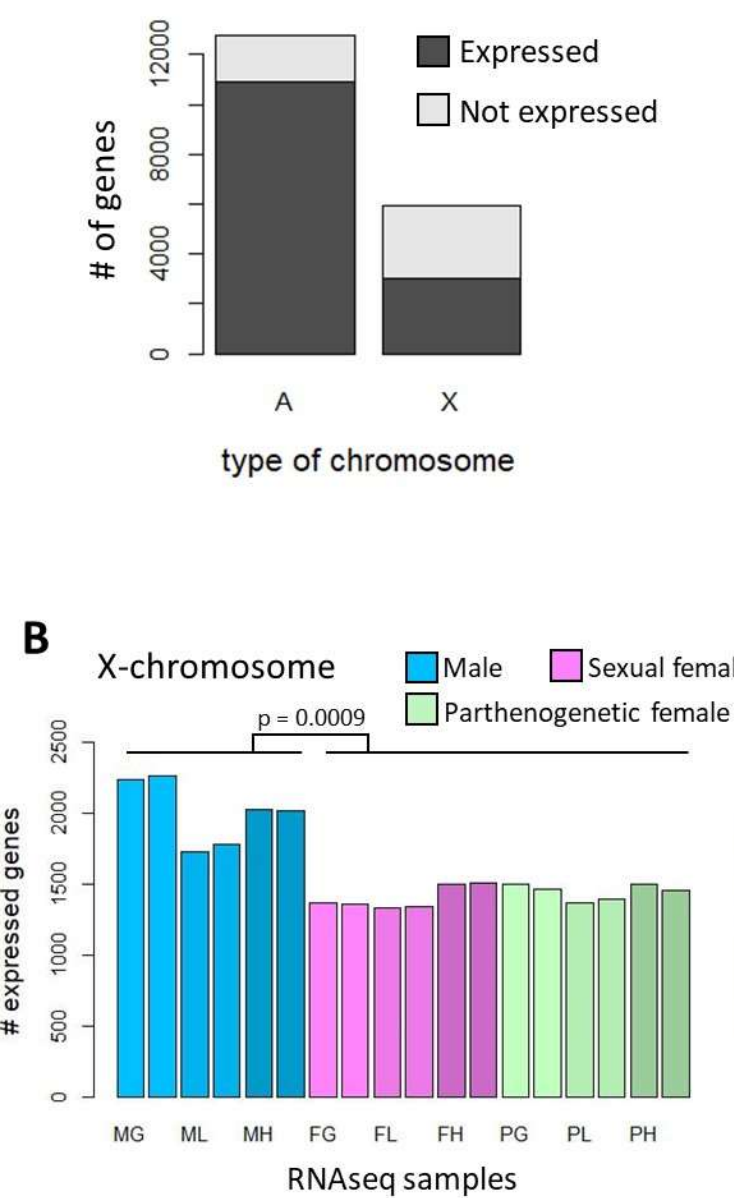

C

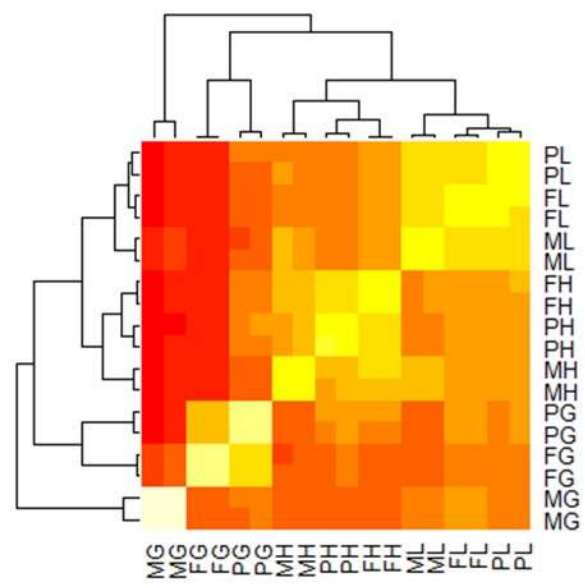

Figure 1. Gene expression in the 18 different RNA-seq samples. A) Number of genes considered as expressed (CPM>1 in at least two samples) and not expressed on the $\mathrm{X}$ and on the autosomes. $\mathrm{B}$ ) Number of expressed genes per sample (expressed at more than 1 CPM) for X-linked and autosomal genes (significance of differences between the $\mathrm{X}$ and autosomes was estimated with two-sided Mann-Whitney tests). C) Heatmap of $\log (\mathrm{CPM}+1)$. Samples group by tissue for head $(\mathrm{H})$ and leg $(\mathrm{L})$ samples, with the parthenogenetic $(P)$ and sexual female $(F)$ samples being always more similar compared to the male samples (M). Expression patterns of male gonad samples (MG) are the most divergent. MG: male gonad, MH: male head, ML: male leg, FG: sexual female gonad, FH: sexual female head, FL: sexual female leg, PG: parthenogenetic female gonad, PH: parthenogenetic female head, PL: parthenogenetic female leg. 


\section{The $X$ chromosome is enriched in male-biased genes at the tissue-level}

To test whether some masculinization of the $X$ was observed at the tissue-level, we first categorized genes according to their relative expression patterns in the different conditions. We defined a gene as "biased" toward, or preferentially expressed in, a set of samples (which can be a particular tissue from a particular morph, all the tissues from a particular morph or a particular tissue in all morphs) when at least $70 \%$ of all reads mapping to this gene were observed in this set of samples (see methods). Testes showed the highest number of biased genes, with 1170 genes (referred to as MG+ genes) being preferentially expressed in this tissue. Then came sexual female ovaries, with $375 \mathrm{FG}+$ genes, and male heads, with $203 \mathrm{MH}+$ genes (figure 2). Tissue-biased genes (i.e., genes expressed mainly in a tissue of all morphs) were common. Heads showed the highest number of tissue-biased genes (1169 H+ genes), followed by legs (607 L+ genes) and gonads (511 G+ genes). Contrastingly, morph-biased genes (i.e., genes expressed mainly in a morph in all tissues) were much less frequent for females (only $58 \mathrm{~F}+$ and $130 \mathrm{P}+$ genes) than for males (596 M+ genes) (figure 2). When considering all genes that were preferentially expressed in a given morph, without considering tissues (e.g., $\mathrm{MG}+, \mathrm{MH}+, \mathrm{ML}+$ and $\mathrm{M}+$ genes for males), a total of 2001, 501 and 299 genes were biased toward males, sexual females and parthenogenetic females, respectively.

Interestingly, these different categories of genes differed in their chromosomal locations (figure 2, Supplementary table S2). The proportions of X-linked genes among genes expressed preferentially in testes $(\mathrm{MG}+)$, male heads $(\mathrm{MH}+)$, male legs $(\mathrm{ML}+)$ or simply in males regardless of tissue $(\mathrm{M}+)$ varied from $54 \%$ to $73 \%$, and significantly exceeded (two-sided binomial tests, $p<10^{-7}$ in all cases) the null expectation, which we took as the proportion of X-linked genes among all expressed genes (22\%) (if we consider all predicted genes - supported by expression data or not $-31.8 \%$ locate on the $\mathrm{X}$ ).

Contrastingly, genes that were preferentially expressed in parthenogenetic females were less likely to locate on the $X$ than expected (two-sided binomial tests, $p$ ranging from 0.014 to 0.023 for $\mathrm{PG}+, \mathrm{PH}+$ and $\mathrm{P}+$, not significant for $\mathrm{PL}+$ ), with proportions of $\mathrm{X}$-linked genes ranging from $0 \%$ to $13 \%$ depending on tissues. The proportion of $X$-linked genes among sexual female-biased genes were intermediate (13\% to $32 \%)$, with only those preferentially expressed in sexual female gonads being more frequent on the $\mathrm{X}$ (two-sided binomial test, $p$ 
$\left.=10^{-5}\right)$. Genes that were preferentially expressed in gonads $(G+)$ showed no deviation from the null expectation, as $22 \%$ of them located on the $\mathrm{X}$, while genes preferentially expressed in heads $(H+)$ and in legs $(L+)$ were significantly less frequent on the $X(15 \%$ to $16 \%)$ than expected (two-sided binomial tests, $p=10^{-8}$ and $p=0.0006$, respectively).

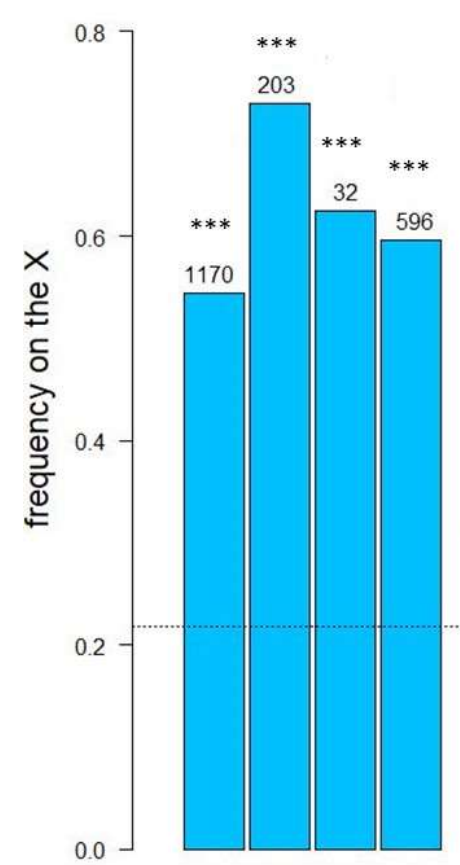

$M G+M H+M L+M+$

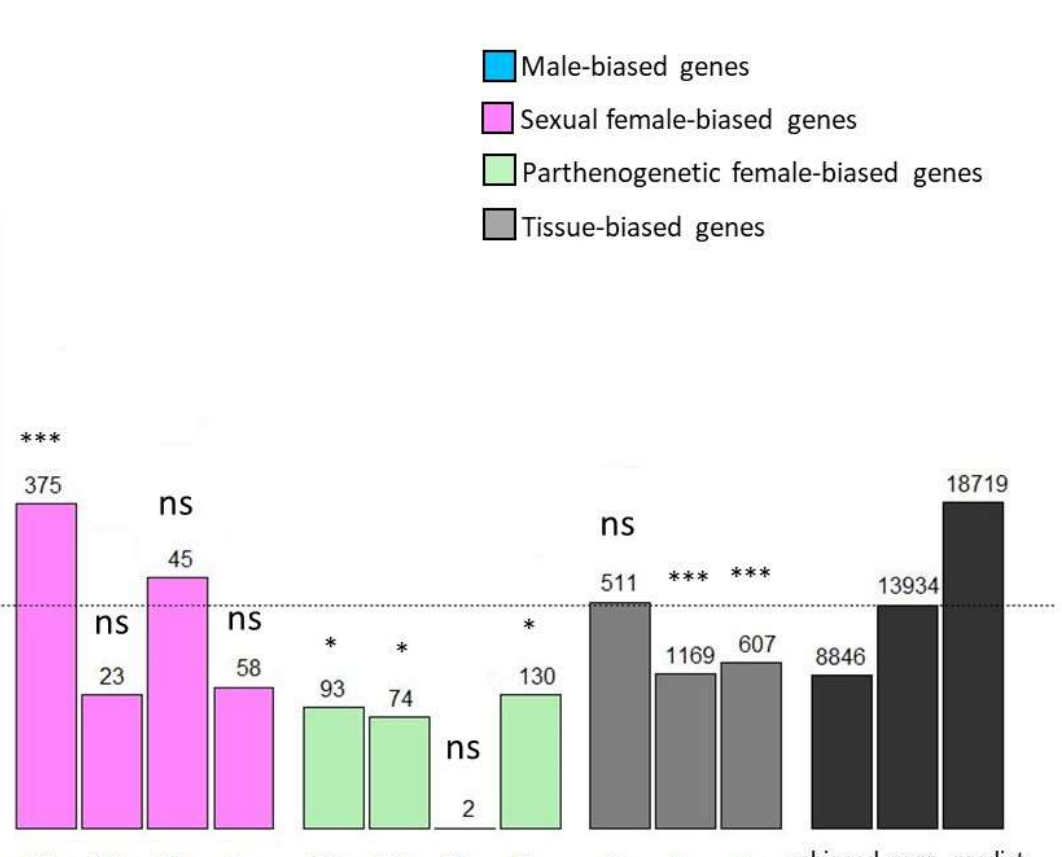

$\mathrm{FG}+\mathrm{FH}+\mathrm{FL}+\mathrm{F}+\mathrm{PG}+\mathrm{PH}+\mathrm{PL}+\mathrm{P}+$
G+ $\quad H+\quad L+$ unbiased expr. predict.

Gene expression patterns

Figure 2. Proportions of $X$-linked genes among genes preferentially expressed in various morphs and/or tissues. Blue bars represent male-biased genes; $M G+, M H+$ and $M L+$ : genes expressed preferentially in male gonads, heads and legs, respectively. $M+$ : genes preferentially expressed in males when pooling all tissues, excluding the genes assigned to the previous categories. Pink bars represent sexual female-biased genes, with $F$ standing for females and letters $G, H$ and $L$ having the same meaning as in males. Green bars represent parthenogenetic female-biased genes (P). Grey bars represent genes expressed preferentially in one of the tissues (gonads, heads or legs) and not limited to a particular morph. Black bars represent the frequency of X-linked genes among genes with unbiased expression ("unbiased"), genes expressed with CPM $>1$ in at least two libraries ("expr.") or all predicted genes ("predict."). The horizontal dotted line represents the proportion of X-linked gene among expressed genes. The number of genes from each category is shown above bars, as well as the $\mathrm{p}$-value (two-sided binomial tests against the expected frequency on the $\mathrm{X}$ chromosome estimated from expressed genes, which corresponds to the dotted horizontal black line). ${ }^{* *}: p<0.001 ; * *: p<0.01 ; *: p<0.05 ; \mathrm{ns}: p \geq 0.05$. 


\section{Expression patterns of single- and two-copy genes}

To investigate the extent to which gene duplication facilitates the evolution of gene expression toward the sex-specific optimum, we compared the expression of autosomal and X-linked genes that belong to single-copy and multicopy gene families. Multigenic gene families were identified by Boulain et al. (2018) from orthoDB on 17 arthropod genomes.

We found that the $\mathrm{X}$ chromosome contained more genes that belong to multicopy families than autosomes ( $38 \%$ of the genes on the $\mathrm{X}$ belong to multicopy families, against $28 \%$ for autosomal genes). When restricting our analyses to genes supported by expression data, 1633 and 7428 single-copy genes locate on the $X$ and on autosomes, respectively. We also found 210 gene families composed of two expressed genes with one being on the $X$ and the other on autosomes. On autosomes, the percentages of genes with male-biased expression (combining $\mathrm{M}+, \mathrm{MG}+, \mathrm{ML}+$ and $\mathrm{MH}+$ genes) were very similar between singleand two-copy genes, at $6.5 \%$ and $6.6 \%$ respectively (figure 3 , Chi-squared test, $\chi 2 \approx 0, p=1$ ). On the $\mathrm{X}$ chromosome however, the proportion of male-biased genes was significantly higher for two-copy genes (40.5\%) than for single-copy genes (32.5\%) (Chi-squared test, $\chi 2=$ $5, p=0.025)$, these two proportions being much higher than their equivalents on autosomes (Chi-squared tests, single-copy genes: $\chi 2=939.5, p<10^{-15}$, two-copy genes: $\chi 2=64.8, p<$ $\left.10^{-15}\right)$. Sexual female- and parthenogenetic female-biased genes accounted only for a few percent of single- and two-copy genes. These female-biased genes showed minor differences in proportion between chromosomes (significant for single-copy genes only for parthenogenetic female [ $\chi 22=4.9, p=0.027]$ and for sexual female $[\chi 2=8.9, p=0.003]$, figure 3). They constituted similar proportions of the single- and two-copy genes on a given chromosome type (Supplementary table S3). 


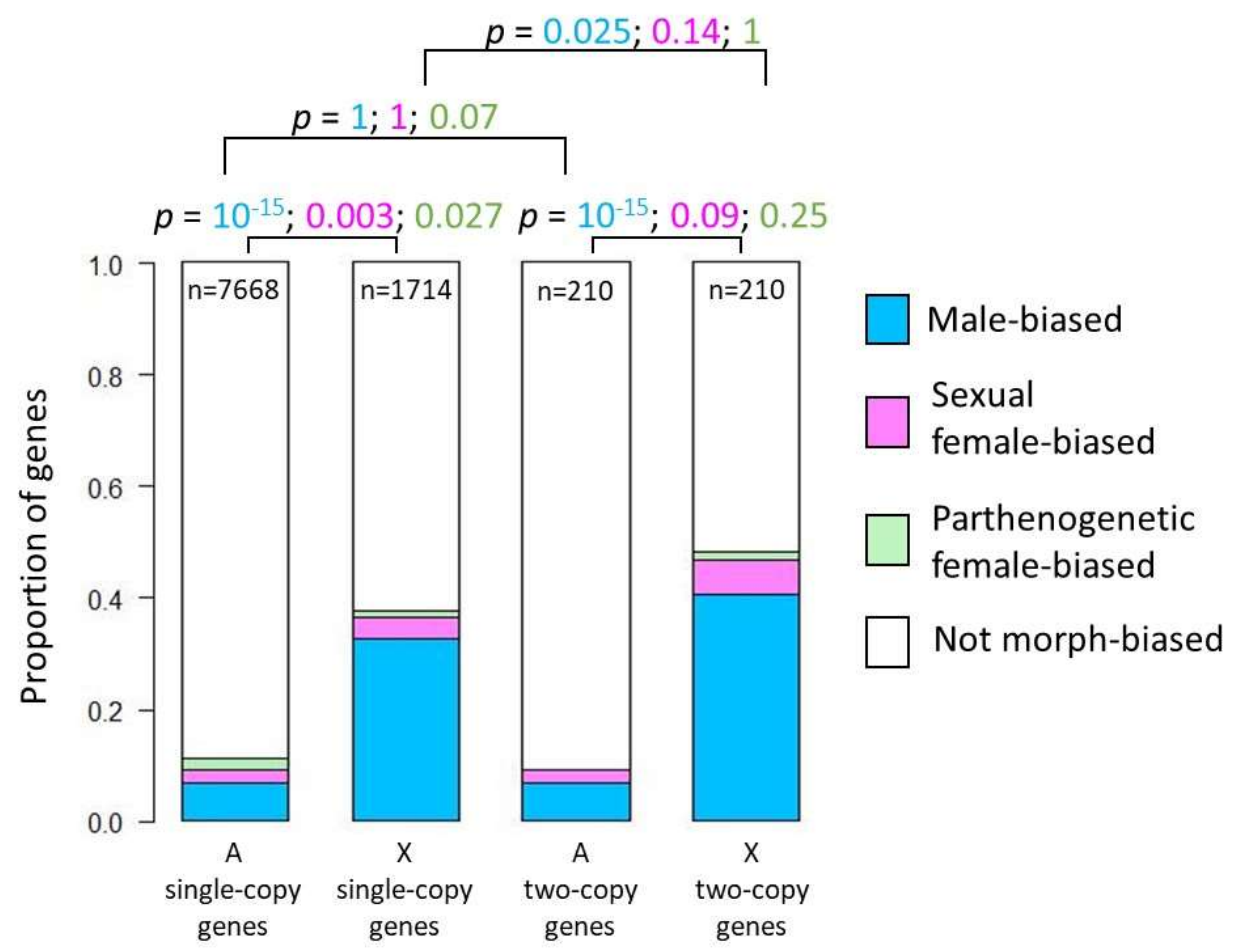

Figure 3. Proportion of genes showing preferential expression in different morphs, according to their number of copies (single or two copies) and chromosomal location (A: autosomes, $\mathrm{X}: \mathrm{X}$ chromosome). Each two-copy gene has one copy on an autosome and the other on the X. P-values (Chi-squared tests) are shown, with font colors corresponding to the tested morph, according to the color of sectors. The number of genes composing each distribution is indicated on the plots.

\section{Expression levels in morphs and tissues}

The median expression levels of $\mathrm{X}$-linked genes in somatic tissues and gonads from male and sexual female morphs were systematically lower than those of autosomal genes (figure $4, p$ $<10^{-15}$ in all comparisons, two-sided Mann-Whitney tests), irrespective of the dose of $X$ chromosomes per cell (two for sexual females and one for males). The same patterns were observed in parthenogenetic females ( $p<10^{-15}$ in all comparisons, supplementary figure S2). The mode of Log2 ratio of male-to-female $\log 2(\mathrm{RPKM}+1)$ (whether sexual or parthenogenetic, figure 4CFI and Supplementary figure S2) lies close to 0 for both autosomal and X-linked genes, indicating dosage compensation for non-sex-biased genes in gonads and somatic tissues. Yet, we observed an excess of genes with high Log2 ratio of male to female 
expression, especially for the $\mathrm{X}$ chromosome in gonads and heads (figure 4CFI). This indicates an overexpression of some of the genes located on the single $\mathrm{X}$ chromosome of male cells, which exceeds dosage compensation. This pattern was expected, given that male-biased genes are significantly more frequent on the $X$ than on autosomes (figure 2).
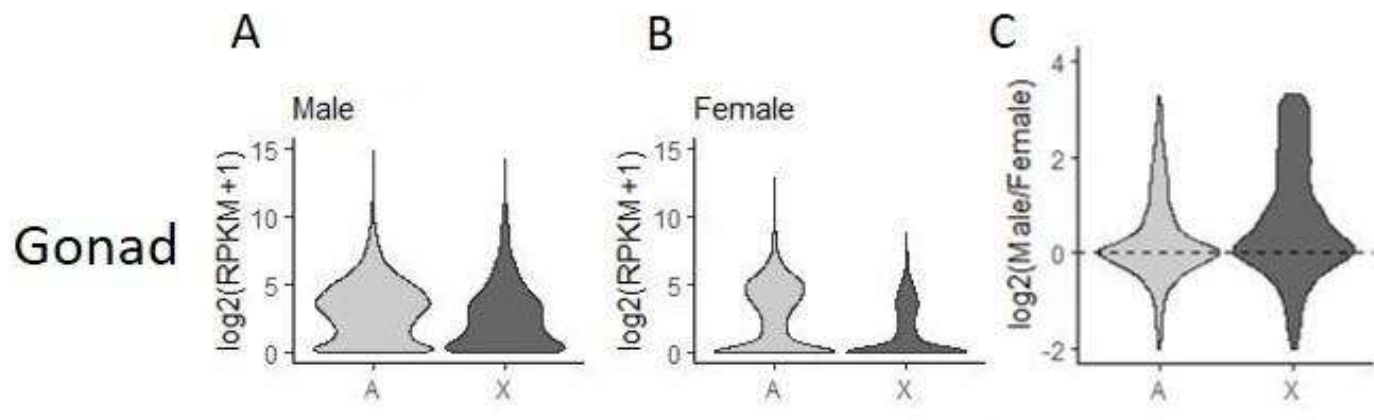

D

$\mathrm{E}$
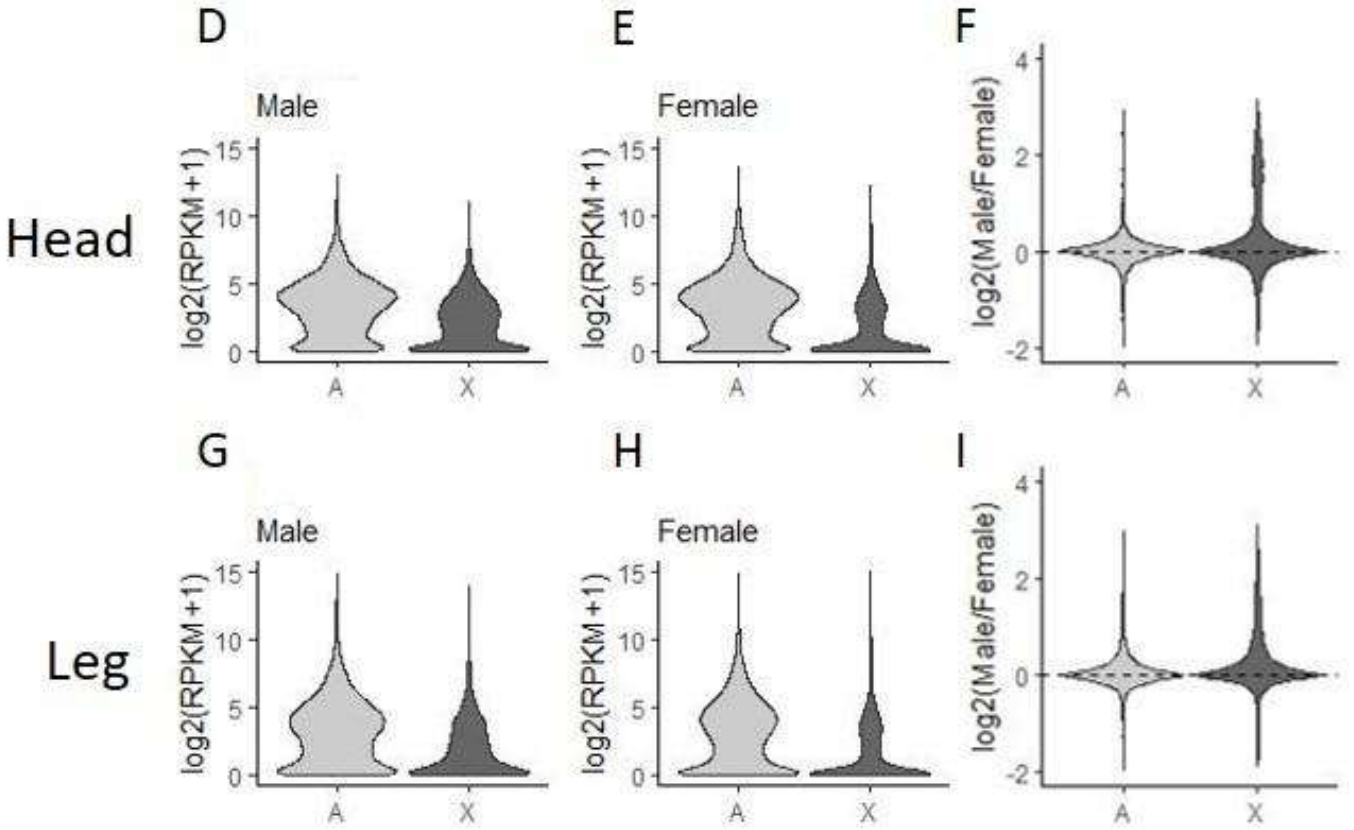

Figure 4. Logarithm of gene expression (RPKM, reads per kilobase per million mapped reads) for Xlinked and autosomal genes for the different types of libraries (gonads, heads or legs of males and sexual females). X-linked genes are significantly less expressed than autosomal genes in all cases (two-sided Mann-Whitney tests, $p<10^{-15}$ ). Logarithm of male-to-sexual female ratio of $\log 2(R P K M+1)$ is also shown for the three different tissues. 


\section{Discussion}

Because of the peculiar inheritance of the $\mathrm{X}$ chromosome in aphids - males transmit systematically their unique $X$ to all sperm cells leading to the production of female-only progeny - , the presence of three distinct morphs (sexual females, parthenogenetic females and males) and the alternation between sexual and asexual reproduction, a specialization of the $X$ into male functions is expected (Jaquiéry et al. 2013). Indeed, models have shown that the conditions for invasion by male-beneficial/parthenogenetic female-detrimental SA alleles are less restrictive for the $X$ than for autosomes, while the opposite is true for maledetrimental/parthenogenetic female-beneficial alleles. SA alleles that are favorable to sexual females should show little bias, the direction of which (i.e., the depletion or enrichment of the $X$ with genes carrying such variants) depending on the selective effect of the allele on males and parthenogenetic females (table 1). A key finding of these predictions is that they are not qualitatively affected by allele dominance level $h$ (the aphid $X$ chromosome is a preferred location for male-beneficial alleles for all values of $h \neq 1$ ). This contrasts with other $X 0$ and $X Y$ species, where the $X$ accumulates both recessive $(h<0.5)$ male-beneficial alleles and dominant $(h>0.5)$ female-beneficial alleles (Vicoso and Charlesworth 2006; Ellegren and Parsch 2007). Consequently, simpler predictions can be made on aphids: the $X$ should be enriched with male-beneficial alleles, parthenogenetic female-beneficial alleles should be more common on autosomes (being counter selected on the $X$ ), and sexual-female beneficial alleles should show no consistent bias (table 1). 
Table 1. Preferred chromosomal location (X chromosome versus autosomes) of different types of alleles with morph-antagonistic effects $\left(s_{m}, s_{f}\right.$ and $s_{p}$ stand for the selective coefficient of the new variant on males, sexual females and parthenogenetic females, respectively). Predictions originate from analytical and simulation models developed in Jaquiéry et al. (2013).

\begin{tabular}{|l|l|}
\hline $\begin{array}{l}\text { Fitness effect of a } \\
\text { SA mutation }\end{array}$ & $\begin{array}{l}\text { Preferred chromosomal location in } \\
\text { aphids }\end{array}$ \\
\hline$s_{m}>0, s_{f}<0, s_{p}<0$ & Favored if on the X \\
\hline$s_{m}>0, s_{f}>0, s_{p}<0$ & Favored if on the X \\
\hline$s_{m}<0, s_{f}<0, s_{p}>0$ & Disfavored if on the X \\
\hline$s_{m}<0, s_{f}>0, s_{p}>0$ & Disfavored if on the X \\
\hline$s_{m}<0, s_{f}>0, s_{p}<0$ & Slightly disfavoured if on the X \\
\hline$s_{m}>0, s_{f}<0, s_{p}>0$ & Slightly favoured if on the X \\
\hline
\end{tabular}

Although high-throughput approaches have been developed to pinpoint putative SA genes (Innocenti and Morrow 2010; Lucotte et al. 2016; Ruzicka et al. 2019), identifying these genes and estimating sex-specific selection and dominance coefficients are challenging tasks that have been achieved in only a handful of studies (Barson et al. 2015; Husby et al. 2015). However, since the evolution of a lower expression level in the sex that suffers from an antagonistic allele could help to resolve intra-locus sexual conflicts and allow the SA allele to reach fixation (Rice 1984; Vicoso and Charlesworth 2006; Ellegren and Parsch 2007), the chromosomal location of sex-biased genes can be used to indirectly test predictions.

Here, we analyzed gene expression in different tissues of distinct morphs in the pea aphid and found that the chromosomal location of morph-biased genes followed the predictions made under SA models from Jaquiéry et al. (2013). In the three tissues considered, male-biased genes were largely overrepresented on the $X$, parthenogenetic female-biased genes were underrepresented, and sexual female-biased genes showed no consistent bias. These empirical data thus support the hypothesis that, in aphids, sexual conflicts would be the key driver of the masculinization of the $X$ and of the specialization of autosomes for the parthenogenetic phase of the life cycle. 
Genes showing a male-biased expression were much more frequent on the $X$ chromosome than expected under a random distribution, irrespective of the tissue considered. Genes expressed mainly in testes, male heads and male legs were 2.5 to 3.5 times more frequent than expected on the $X$, so were genes that were male-biased in all the tissues considered. As all the investigated tissues contribute to this pattern, these results extend previous studies reporting a general enrichment in male-biased genes (measured from whole-body samples) on the aphid X chromosome (Jaquiéry et al. 2013; Jaquiéry et al. 2018, Mathers et al. 2019). Overall, this and previous studies demonstrate strong and consistent bias toward the $\mathrm{X}$ in aphids for genes expressed predominantly in males. Interestingly, on the $\mathrm{X}$ chromosome, the percentage of male-biased genes was significantly higher for two-copy genes (40.5\%) than for single-copy genes (32.5\%), while these percentages were similar for autosomal genes (6.5\% and 6.6\%). These results match the predictions that duplications may facilitate sub- or neo-functionalization toward sex-specific optima, and that, for genes located on the $\mathrm{X}$ chromosome, the male optimal expression levels are favored rather than the female optima.

Conversely, parthenogenetic female-biased genes were significantly less frequent on the $\mathrm{X}$, except for $\mathrm{PL}+$ genes due to lack of power, as only two such genes were found, both located on autosomes. This matches previous observations on whole body transcriptomes (Jaquiéry et al. 2013; Mathers et al. 2019). This trend also reveals a consistent reduction of contribution of the $\mathrm{X}$ chromosome to biological functions and processes occurring in parthenogenetic females, and corroborates the observation that the $\mathrm{X}$ is depleted from functionally important genes for the parthenogenetic phase (Li et al. 2020). Interestingly, the chromosomal location of sexual female-biased genes did not significantly depart from random expectations, except for genes specifically expressed in ovaries. These were significantly more frequent than expected on the $X$, although their proportion (32\%, Figure 2) was much lower than for male-biased genes (54\%-73\%). According to the models (table 1), variants of $X$-linked genes that are beneficial to sexual females should increase in frequency only if they are also beneficial to males. This could be the case for genes controlling sexual reproduction-related functions that were not sex-specific (e.g., meiosis). In this case however, we do not expect such genes to be preferentially expressed in sexual females. These genes may be less/not expressed in adult males because spermatogenesis is 
often already completed at this stage (Wieczorek et al. 2019). Alternatively, these genes used to have variants beneficial to both sexes, which have since evolved functions that are more specific to sexual-females.

Among hemipterans, the accumulation of male-biased genes on the $X$ appears to be specific of aphids. A deficit of male-biased genes on the $X$ was observed in three hemipteran species (two heteropteran bugs and a leafhopper) that only reproduce sexually and are very distantly related to aphids, despite an apparent homologous origin of the $\mathrm{X}$ chromosome (Pal and Vicoso 2015; Mathers et al. 2020). In psyllids, which diverged more recently ( 200250 MYA) from aphids and which are characterized by obligate sexual reproduction and X0 sex determination, no enrichment of male-biased genes on the $X$ chromosome was found (Li et al. 2020). Hence, the accumulation of male-biased genes on the $X$ chromosome in aphids would have started after the divergence between aphids and psyllids. This scenario supports a role for cyclical parthenogenesis (which appeared 200 MYA in aphid ancestors, Davis 2012) in the masculinization of the $X$ in aphids.

While the chromosomal location of the different types of sex-biased genes matches the predicted evolution of gene expression as a mitigation mechanism of sexual conflicts, other processes could contribute to the observed patterns. Genes located on different chromosomes types could differ in other characteristics that could result in a non-random genomic distribution of male-biased genes. For example, the $\mathrm{X}$ chromosome of Drosophila is enriched in young genes (those that are present in only a restricted taxonomic group), which are more likely to show sex-biased expression (Palmieri et al. 2014). In our analysis however, the X-linked copy of a two-copy gene had a much greater probability of being male-biased $(0.405)$ than its autosomal counterpart $(0.066$, figure 3$)$, demonstrating that the masculinization of the $X$ does not solely reflect differences in gene characteristics.

Moreover, the three $A$. pisum autosomes are systematically depleted in male-biased genes and enriched in parthenogenetic female-biased genes (supplementary table S4). This reinforces the specific hemizygosity of the $X$ in males as a determinant factor for the observed patterns. Consequently, we do not see an alternative hypothesis to explain the accumulation of male-biased genes on the $X$ to the one based on the resolution of sexual conflicts by an evolution of sex-specific or sex-biased gene expression. 
The number of morph-biased genes was highest for gonadic tissues, with 1170, 375 and 130 genes being specific of gonads of males, sexual females or parthenogenetic females, respectively. This observation likely reflects the highly morph-specific functions of gonads: the production of sperm in males, of yolky eggs in sexual females and of embryos in viviparous parthenogenetic females (Michalik et al. 2013). In other species, testis also stands out as the tissue showing the most specific gene expression patterns (Meiklejohn and Presgraves 2012; Uhlén et al. 2015). Many genes also showed preferential expression in male heads (202), against 23 and 93 for sexual and parthenogenetic female heads, respectively. This could reflect sensorial and/or behavioral differences between males and females: males have to actively search for females and initiate mating, while females spend most of their time in feeding. Legs showed a low number of morph-biased genes, most of which were overexpressed in sexual females (45 genes, against 32 for males and 2 for parthenogenetic females). This result may reflect the existence of specific organs on sexual female tibias (scent plaques), which are responsible for the secretion and release of sex pheromones (Murano et al. 2018).

Interestingly, the strong enrichment of the $X$ with male-biased genes is similar across tissues (ranging from 54\% to 73\%). This result strikingly contrasts with Drosophila, where male-biased genes from different tissues show opposing patterns: male-biased genes in brains are strongly enriched on the $X$, while there is either no departure from random expectation or a paucity of male-biased genes on the $\mathrm{X}$ for the other tissues (Huylmans and Parsch 2015). These differences were interpreted as resulting from the interplay between dosage compensation (the brain could be more sensible to gene dose, and thus would require a tighter dosage control) and sex-specific regulation of gene expression (Huylmans and Parsch 2015). In aphids, the consistent enrichment of the $X$ with male-biased genes in different tissues suggests that similar evolutionary forces apply in reproductive and somatic tissues. Under the hypothesis that sex-biased expression evolved in response to sexual conflicts, our results suggest that their attenuation occurs in all tissues. Our data also indicate that sexual conflicts occur in a wide range of tissues and not only in gonads. This pattern was observed by Innocenti and Morrow (2010), who found that transcripts showing signature of sexual antagonism in Drosophila were frequent in soma. 
Previous studies suggested dosage compensation in the pea aphid from whole-body transcriptomes (Jaquiéry et al. 2013; Pal and Vicoso 2015; Li et al. 2020). An increased accessibility of the chromatin of the $X$ in males (Richard et al. 2017) and a specific pattern of DNA methylation on the $X$ for sex-biased genes (Mathers et al. 2019) were also documented. Here, we show dosage compensation in all investigated tissues, including testis. While further work is required to demonstrate that X-linked genes are dosage compensated in various cell types during spermatogenesis, dosage compensation in testis would be another peculiarity of aphids. Indeed, sex chromosomes of other dosagecompensated species are generally not compensated in the gonads of the heterogametic sex in diptera and lepidoptera (Vicoso and Bachtrog 2015; Gu et al. 2017; Gu and Walters 2017). In other groups (mammals, birds, nematods, fungi), sex-linked genes are even silenced by meiotic sex chromosome inactivation (Shiu et al. 2001; Bean et al. 2004; Turner 2007; Schoenmakers et al. 2009; but see Guioli et al. 2012; Daish et al. 2015). The silencing of sex-linked genes during gametogenesis could have evolved to protect from the invasion of segregation distorters that would bias sex-ratio (Meiklejohn and Tao 2010). By contrast, segregation distortion cannot affect the aphid X chromosome during spermatogenesis, as all sperm cells that do not carry an $X$ degenerate. That all aphid sperm cells carry one $X$ copy could explain why the expression of X-linked genes in aphid testis has not been repressed during the course of evolution, and thus can be subject to dosage compensation.

In conclusion, we document an atypical genome-wide pattern of gene expression in aphids, with a high degree of masculinization of the $\mathrm{X}$ chromosome in both somatic and gonadic tissues. Our study reinforces the hypothesis that this masculinization evolved in response to sexual conflicts raised by the accumulation of male-beneficial alleles on the $X$ (Jaquiéry et al. 2013). To further support this hypothesis, masculinization of the $X$ should be assessed in distantly related aphid lineages (which diverged up to 200 MYA, Davis 2012) and other species that show an "aphid-like" life cycle and X-inheritance (e.g. Strongyloides nematods, Nemetschke et al. 2010; Streit 2017). Finally, understanding the functional epigenetic or post-transcriptional mechanisms responsible for sex-biased gene expression in aphids would help to understand how such a strong chromosomal specialization in gene expression has been achieved. 


\section{Methods}

\section{Sex- and tissue-biased gene expression analysis}

Gene expression levels in three different tissues (head, legs, gonads) of three reproductive morphs (male, sexual female, parthenogenetic female) were measured from RNA-Seq collected on a single pea aphid genotype (clone LSR1, from alfalfa, IAGC 2010). Aphids were reared on broad bean Vicia faba at less than five individuals per plant to prevent the production of winged morphs. Parthenogenesis was maintained under a 16-hour light regime and a temperature of $18^{\circ} \mathrm{C}$. The production of sexual individuals was initiated by transferring larvae (at stage 3) to a 12-hour light regime at the same temperature of $18^{\circ} \mathrm{C}$. Two generations later, sexual females and males were observed. A total of 100 adult parthenogenetic females (produced under 16-hour light regime), 100 adult sexual females and 100 adult males were immediately frozen into liquid nitrogen. Heads and legs were scalpel-cut. Twenty additional individuals per morph were also dissected in a saline solution with fine forceps to collect gonads. Gonads included testes and accessory glands in males and ovarioles in sexual females. Embryos of stage $>10$ (according to Miura et al. 2003) were removed from parthenogenetic female ovarioles (which already contain asexually developing embryos) to avoid the contribution of developing and late embryos to RNA production. All collected tissues were stored in RNA later (Qiagen) immediately after collection and pooled in batches before RNA extraction (with two replicates by sex and tissue). Hence, a total of 18 RNA extractions ( 3 morphs $\times 3$ tissues $\times 2$ biological replicates) were performed using the SV Total RNA Isolation System (Promega) according to manufacturer's instructions. RNA quality was checked on Bioanalyzer (Agilent) and quantified on Nanodrop (Thermo Scientific). The 18 RNA samples were subsequently sent to the GetPlage platform (Toulouse, France) for library preparation (TruSeq Stranded mRNA Library Preparation kit) and 150 bp RNA paired-end sequencing (Illumina HiSeq3000).

After filtering for rRNA, reads from each library were mapped to the V2 assembly of the pea aphid genome (Acyr 2.0, Genbank accession GCA_000142985.2) using STAR version 2.5.2a (Dobin et al. 2013) with default parameters, except: outFilterMultimapNmax = 5, outFilterMismatchNmax = 3, alignIntronMin = 10, alignIntronMax $=50000$ and alignMatesGapMax $=50000$. Then, we counted reads mapped on exons of each predicted 
gene (NCBI Annotation release ID: 102) using FeatureCounts version 1.5.0-p3 (Liao et al. 2014) with default parameters except: -g gene -C - $p$-M --fraction. The numbers of mapped reads per library ranged from 19.8 to 28.6 million (mean 24.3 million, supplementary table S1).

We used the R package DESeq (Anders and Huber 2010) to normalize the libraries (upperquartile method with $p=0.75$ ) and calculate CPM. Only genes with CPM>1 in at least two libraries (out of 18) were considered as expressed and retained in the analyses, unless mentioned otherwise. To identify genes predominantly expressed in a specific tissue or a morph, we imposed that a minimal percentage $(70 \%)$ of the reads mapping to a given gene was sequenced from that specific tissue/morph. Doing so avoided the reliance on $\mathrm{p}$-values from differential expression analyses (which in turn depend on the absolute expression level of a gene). This $70 \%$ threshold allowed identifying genes showing considerable bias in expression toward a tissue/morph, and to retrieve a large number of genes for more powerful analyses. So, when $>70 \%$ of the reads mapping to a gene were from a given tissue from a given morph, the gene was classified as predominantly expressed in that tissue (e.g., $\mathrm{PL}+$ for those predominantly expressed in legs of parthenogenetic females; etc). When $>70 \%$ of the reads were from a given morph (but not restricted to a single tissue), the gene was classified as morph-specific ( $\mathrm{M}+, \mathrm{F}+$ or $\mathrm{P}+$, for males, sexual females, or parthenogenetic females, respectively). Similarly, genes mainly expressed ( $>70 \%$ of reads) in a tissue (but not restricted to a single morph) were classified as tissue-specific ( $\mathrm{G}+, \mathrm{H}+$ or $\mathrm{L}+$, for gonads, heads or legs, respectively). Expressed genes were thus classified into 16 mutually exclusive classes: $\mathrm{MH}+, \mathrm{ML}+, \mathrm{MG}+, \mathrm{FH}+, \mathrm{FL}+, \mathrm{FG}+\mathrm{PH}+, \mathrm{PL}+, \mathrm{PG}+, \mathrm{M}+, \mathrm{F}+, \mathrm{P}+, \mathrm{H}+, \mathrm{L}+, \mathrm{G}+$ or unbiased.

\section{Comparisons between chromosome types}

The chromosomal location (X vs autosomes) of each gene was that of Jaquiéry et al. (2018). This represented the only assignments available at the start of the analyses. Since then, chromosome-level genome assemblies have been produced for the pea aphid (Li et al. 2019; Mathers et al. 2020). We found that our assignments to chromosomes were consistent for $97 \%$ to $98 \%$ of the genes located on the four mega scaffolds corresponding to the four pea aphid chromosomes, depending on the assembly. We therefore considered our initial assignment reliable. Furthermore, the number of NCBI predicted genes that we assigned to 
chromosome (18719) was larger than for the Li et al. (2019) assembly (17315). For the genome assembly from Mathers et al. (2020), DNA was obtained from a linage sampled on Lathyrus, a host plant genus that harbors a cryptic species of the pea aphid complex (Peccoud et al. 2009; 2014) that is quite divergent from the LSR1 clone we used (J. Peccoud, personal communication). Significant deviation from a random chromosomal distribution for each class of genes with a specific expression pattern was tested with two-sided binomial tests, the expected proportion was computed as the proportion of expressed genes on the $\mathrm{X}$. To compare expression patterns between the three autosomes (supplementary table S4), we used the assembly from Li et al. (2019).

\section{Investigation of gene families}

To identify single-copy and multicopy genes in A. pisum genome, we used orthologs identified among 17 arthropod genomes by Boulain et al. (2018). Briefly, the longest protein isoform from each arthropod species was used to run OrthoDB_soft_1.6 (Kriventseva, et al. 2015) and the levels of orthology were assigned by referring to the species phylogeny established in Boulain et al. (2018). The groups of orthologs generated by OrthoDB were then used to identify A. pisum unique (single-copy) or duplicated (multicopy) genes. To control for possible differences in gene content between the $X$ and autosomes, which could account for different expression patterns between chromosomes, we searched for gene families composed of two genes, one being on the $X$ and the other autosomes. Given the low number of genes satisfying this criterion ( $n=210)$, gene classes with male-biased expression (i.e., M+, MG+, ML+ and MH+ genes) were aggregated. We proceeded similarly for sexual female-biased genes and parthenogenetic female-biased genes. For each aggregated class of morph-biased gene, we compared its frequency among chromosomes for single and duplicated copies and then among single and duplicated copies within chromosomes (chi-squared tests).

\section{Dosage compensation}

To investigate dosage compensation, RPKM (reads per kilobase per million mapped reads) were calculated with EdgeR (Robinson et al. 2010), and only genes with RPKM > 1 in at least two of the 18 libraries were kept. After log-transformation of RPKM, differences in expression levels between chromosomes were examined with two-sided Mann-Whitney 
tests for each sex and each tissue. Then the logarithm of the male to female (sexual or parthenogenetic) ratio of $\log 2(\mathrm{RPKM}+1)$ were estimated for $\mathrm{X}$ and autosomes for each tissue.

\section{Acknowledgments}

This work was supported by a grant from the University of Rennes 1 ("Défi émergent") to JJ, as well as grants from the French Research Agency (ANR) SexAphid (ANR-09-GENM-017001) and Mecadapt (ANR-11-BSV7-005-01). The authors declare no conflicting interest. The raw data will be publicly available on NCBI (projects PRJNA547535 and PRJNA748848) once the article will be accepted. 


\section{References}

Anders S, Huber W. 2010. Differential expression analysis for sequence count data. Genome Biology 11:R106.

Arthur RK, Ma L, Slattery M, Spokony RF, Ostapenko A, Nègre N, White KP. 2014. Evolution of H3K27me3-marked chromatin is linked to gene expression evolution and to patterns of gene duplication and diversification. Genome research 24:1115-1124.

Barson NJ, Aykanat T, Hindar K, Baranski M, Bolstad GH, Fiske P, Jacq C, Jensen AJ, Johnston SE, Karlsson S, et al. 2015. Sex-dependent dominance at a single locus maintains variation in age at maturity in salmon. Nature 528:405-408.

Bean CJ, Schaner CE, Kelly WG. 2004. Meiotic pairing and imprinted X chromatin assembly in Caenorhabditis elegans. Nature Genetics 36:100-105.

Blackman RL. 1987. Reproduction, cytogenetics and development. In: Minks A, P H, editors. Aphids: their biology, natural enemies and control. Amsterdam: Elsevier. p. 163-195.

Bonduriansky R, Chenoweth SF. 2009. Intralocus sexual conflict. Trends in Ecology and Evolution 24:280-288.

Boulain H, Legeai F, Guy E, Morliere S, Douglas NE, Oh J, Murugan M, Smith M, Jaquiéry J, Peccoud J, et al. 2018. Fast evolution and lineage-specific gene family expansions of aphid salivary effectors driven by interactions with host-plants. Genome Biology and Evolution 10:1554-1572.

Cheng C, Kirkpatrick M. 2016. Sex-specific selection and sex-biased gene expression in humans and flies. Plos Genetics 12:e1006170.

Chippindale AK, Gibson JR, Rice WR. 2001. Negative genetic correlation for adult fitness between sexes reveals ontogenetic conflict in Drosophila. Proceedings of the National Academy of Sciences 98:1671-1675.

Collet JM, Fuentes S, Hesketh J, Hill MS, Innocenti P, Morrow EH, Fowler K, Reuter M. 2016. Rapid evolution of the intersexual genetic correlation for fitness in Drosophila melanogaster. Evolution 70:781-795.

Connallon T, Clark AG. 2011. The resolution of sexual antagonism by gene duplication. Genetics 187:919-937.

Cox R, Calsbeek R. 2009. Sexually antagonistic selection, sexual dimorphism, and the resolution of intralocus sexual conflict. The American Naturalist 173:176-187.

Daish TJ, Casey AE, Grutzner F. 2015. Lack of sex chromosome specific meiotic silencing in platypus reveals origin of $\mathrm{MSCl}$ in therian mammals. BMC Biology 13:106.

Davis GK. 2012. Cyclical parthenogenesis and viviparity in aphids as evolutionary novelties. Journal of Experimental Zoology Part B: Molecular and Developmental Evolution 318:448-459.

Dean R, Perry JC, Pizzari T, Mank JE, Wigby S. 2012. Experimental evolution of a novel sexually antagonistic allele. Plos Genetics 8:e1002917.

Dobin A, Davis CA, Schlesinger F, Drenkow J, Zaleski C, Jha S, Batut P, Chaisson M, Gingeras TR. 2013. STAR: ultrafast universal RNA-seq aligner. Bioinformatics 29:15-21.

Ellegren H, Parsch J. 2007. The evolution of sex-biased genes and sex-biased gene expression. Nature Reviews Genetics 8:689-698.

Fry JD. 2010. The genomic location of sexually antagonistic variation: some cautionary comments. Evolution 64:1510-1516. 
Gallach M, Betrán E. 2011. Intralocus sexual conflict resolved through gene duplication. Trends in Ecology and Evolution 26:222-228.

Gu L, Walters JR. 2017. Evolution of sex chromosome dosage compensation in animals: a beautiful theory, undermined by facts and bedeviled by details. Genome Biology and Evolution 9:2461-2476.

Gu L, Walters JR, Knipple DC. 2017. Conserved patterns of sex chromosome dosage compensation in the lepidoptera (WZ/ZZ): insights from a moth neo-Z chromosome. Genome Biology and Evolution 9:802-16.

Guioli S, Lovell-Badge R, Turner JMA. 2012. Error-prone ZW pairing and no evidence for meiotic sex chromosome inactivation in the chicken germ line. Plos Genetics 8:e1002560.

Husby A, Kawakami T, Rönnegård L, Smeds L, Ellegren H, Qvarnström A. 2015. Genome-wide association mapping in a wild avian population identifies a link between genetic and phenotypic variation in a life-history trait. Proceedings of the Royal Society B: Biological Sciences 282:20150156.

Huylmans AK, Parsch J. 2015. Variation in the X:autosome distribution of male-biased genes among Drosophila melanogaster tissues and its relationship with dosage compensation. Genome Biology and Evolution 7:1960-1971.

Innocenti P, Morrow EH. 2010. The sexually antagonistic genes of Drosophila melanogaster. Plos Biology 8:e1000335.

International Aphid Genomics Consortium. 2010. Genome sequence of the pea aphid Acyrthosiphon pisum. PLoS Biology. 8:e1000313.

Jaquiéry J, Peccoud J, Ouisse T, Legeai F, Prunier-Leterme N, Gouin A, Nouhaud P, Brisson JA, Bickel $\mathrm{R}$, Purandare $\mathrm{S}$, et al. 2018. Disentangling the causes for faster-X evolution in aphids. Genome Biology and Evolution 10:507-520.

Jaquiéry J, Rispe C, Roze D, Legeai F, Le Trionnaire G, Stoeckel S, Mieuzet L, Da Silva C, Poulain J, Prunier-Leterme N, et al. 2013. Masculinization of the $X$ chromosome in the pea aphid. Plos Genetics 9.

Jaquiéry J, Stoeckel S, Rispe C, Mieuzet L, Legeai F, Simon JC. 2012. Accelerated evolution of sex chromosomes in aphids, an X0 system. Molecular Biology and Evolution 29:837-847.

Kriventseva EV, Tegenfeldt F, Petty TJ, Waterhouse RM, Simão FA, Pozdnyakov IA, loannidis $P$, Zdobnov EM. 2015. OrthoDB v8: update of the hierarchical catalog of orthologs and the underlying free software. Nucleic Acids Research 43:D250-256.

Li Y, Zhang B, Moran NA. 2020. The aphid X chromosome is a dangerous place for functionally important genes: diverse evolution of Hemipteran genomes based on chromosome-level assemblies. Molecular Biology and Evolution 37:2357-2368.

Li YY, Park H, Smith TE, Moran NA. 2019. Gene family evolution in the pea aphid based on chromosome-level genome assembly. Molecular Biology and Evolution 36:2143-2156.

Liao Y, Smyth GK, Shi W. 2014. featureCounts: an efficient general purpose program for assigning sequence reads to genomic features. Bioinformatics 30:923-930.

Lucotte EA, Laurent R, Heyer E, Ségurel L, Toupance B. 2016. Detection of allelic frequency differences between the sexes in humans: a signature of sexually antagonistic selection. Genome Biology and Evolution 8:1489-1500.

Mathers TC, Mugford ST, Percival-Alwyn L, Chen Y, Kaithakottil G, Swarbreck D, Hogenhout SA, van Oosterhout C. 2019. Sex-specific changes in the aphid DNA methylation landscape. Molecular Ecology 28:4228-4241. 
Mathers TC, Wouters RHM, Mugford ST, Swarbreck D, van Oosterhout C, Hogenhout SA. 2020. Chromosome-scale genome assemblies of aphids reveal extensively rearranged autosomes and longterm conservation of the $\mathrm{X}$ chromosome. Molecular Biology and Evolution. 38:856-875.

Meiklejohn CD, Presgraves DC. 2012. Little evidence for demasculinization of the Drosophila X chromosome among genes expressed in the male germline. Genome Biology and Evolution 4:10071016.

Meiklejohn CD, Tao Y. 2010. Genetic conflict and sex chromosome evolution. Trends in Ecology and Evolution 25:215-223.

Michalik A, Szklarzewicz T, Wegierek P, Wieczorek K. 2013. The ovaries of aphids (Hemiptera, Sternorrhyncha, Aphidoidea): morphology and phylogenetic implications. Invertebrate Biology 132:226-240.

Miura T, Braendle C, Shingleton A, Sisk G, Kambhampati S, Stern DL. 2003. A comparison of parthenogenetic and sexual embryogenesis of the pea aphid Acyrthosiphon pisum (Hemiptera: Aphidoidea). Journal of Experimental Zoology part B: Molecular and Developmental Evolution 295:59-81.

Murano K, Ogawa K, Kaji T, Miura T. 2018. Pheromone gland development and monoterpenoid synthesis specific to oviparous females in the pea aphid. Zoological Letters 4:9.

Nemetschke L, Eberhardt AG, Hertzberg H, Streit A. 2010. Genetics, chromatin diminution, and sex chromosome evolution in the parasitic nematode genus Strongyloides. Current Biology 20:16871696.

Nozawa M, Fukuda N, Ikeo K, Gojobori T. 2014. Tissue- and stage-dependent dosage compensation on the neo-X chromosome in Drosophila pseudoobscura. Molecular Biology and Evolution 31:61424.

Pal A, Vicoso B. 2015. The X chromosome of Hemipteran insects: conservation, dosage compensation and sex-biased expression. Genome Biology and Evolution 7:3259-3268.

Palmieri N, Kosiol C, Schlötterer C. 2014. The life cycle of Drosophila orphan genes. eLife 3:e01311.

Peccoud J, de la Huerta M, Bonhomme J, Laurence C, Outreman Y, Smadja CM, Simon J-C. 2014. Widespread host-dependent hybrid unfitness in the pea aphid species complex. Evolution 68:29832995.

Peccoud J, Ollivier A, Plantegenest M, Simon JC. 2009. A continuum of genetic divergence from sympatric host races to species in the pea aphid complex. Proceedings of the National Academy of Sciences of the United States of America 106:7495-7500.

Rice WR. 1984. Sex chromosomes and the evolution of sexual dimorphism. Evolution 38:735-742.

Rice WR, Chippindale AK. 2002. The evolution of hybrid infertility: perpetual coevolution between gender-specific and sexually antagonistic genes. Genetica 116:179-188.

Richard G, Legeai F, Prunier-Leterme N, Bretaudeau A, Tagu D, Jaquiery J, Le Trionnaire G. 2017. Dosage compensation and sex-specific epigenetic landscape of the $\mathrm{X}$ chromosome in the pea aphid. Epigenetics \& Chromatin 10:30.

Robinson MD, McCarthy DJ, Smyth GK. 2010. edgeR: a Bioconductor package for differential expression analysis of digital gene expression data. Bioinformatics 26:139-140.

Ruzicka F, Hill MS, Pennell TM, Flis I, Ingleby FC, Mott R, Fowler K, Morrow EH, Reuter M. 2019. Genome-wide sexually antagonistic variants reveal long-standing constraints on sexual dimorphism in fruit flies. Plos Biology 17:e3000244. 
Schoenmakers S, Wassenaar E, Hoogerbrugge JW, Laven JSE, Grootegoed JA, Baarends WM. 2009. Female Meiotic Sex Chromosome Inactivation in Chicken. Plos Genetics 5:e1000466.

Shiu PK, Raju NB, Zickler D, Metzenberg RL. 2001. Meiotic silencing by unpaired DNA. Cell 107:905916.

Stewart AD, Pischedda A, Rice WR. 2010. Resolving intralocus sexual conflict: genetic mechanisms and time frame. Journal of Heredity 101:S94-S99.

Streit A. 2017. Genetics: modes of reproduction and genetic analysis. Parasitology 144:316-326.

Turner JMA. 2007. Meiotic sex chromosome inactivation. Development 134:1823-1831.

Uhlén M, Fagerberg L, Hallström BM, Lindskog C, Oksvold P, Mardinoglu A, Sivertsson Å, Kampf C, Sjöstedt E, Asplund A, et al. 2015. Tissue-based map of the human proteome. Science 347:1260419.

Vensko SP, Stone EA. 2015. X-to-autosome expression and msl-2 transcript abundance correlate among Drosophila melanogaster somatic tissues. PeerJ. 3:e771-e

Vicoso B, Bachtrog D. 2015. Numerous transitions of sex chromosomes in Diptera. Plos Biology 13:e1002078.

Vicoso B, Charlesworth B. 2006. Evolution on the X chromosome: unusual patterns and processes. Nature Reviews Genetics 7:645-653.

Wieczorek K, Kanturski M, Sempruch C, Świątek P. 2019. The reproductive system of the male and oviparous female of a model organism-the pea aphid, Acyrthosiphon pisum (Hemiptera, Aphididae). PeerJ 7:e7573.

Williams TM, Carroll SB. 2009. Genetic and molecular insights into the development and evolution of sexual dimorphism. Nature Reviews Genetics 10:797-804.

Wilson ACC, Sunnucks P, Hales DF. 1997. Random loss of $X$ chromosome at male determination in an aphid, Sitobion near fragariae, detected using an X-linked polymorphic microsatellite marker.

Genetical Research 69:233-236. 